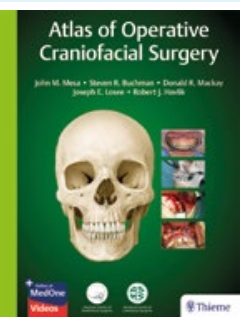

\section{ATLAS OF OPERATIVE CRANIOFACIAL} SURGERY

John Mesa, Steven R. Buchman, Donald MacKay, Joseph Losee, Robert Havlik

2019; Thieme; price $\mathrm{f} 258.50$ pp. 554

ISBN: 9781626236707

This seven-part atlas provides a comprehensive step-by-step surgical guide to craniofacial surgery. Along with the hardback book there is an accessible ebook and videos of surgical procedures for each chapter. Written by leaders in the field of plastic surgery, this book is targeted towards surgical trainees looking for a 'go-to' book when learning steps of particular surgical procedures in the craniofacial region.

Each chapter follows the same format: objective, relevant surgical anatomy, patient selection, indications and contraindications, preoperative planning, surgical instruments, operative procedure, step-by-step technical details, complications, conclusion and surgical pearls. There are plenty of detailed figures and illustrations to complement the text. Each is well referenced for further reading.

Part one is relatively short and discusses 'Fundamental principles' and 'Craniofacial nerve blocks'. Part two dives in to surgery-related to the cranial vault and bony face. This includes management of craniosynostosis and other craniofacial deformities. Common procedures are discussed in good detail with notation to involvement of other specialties such as neurosurgery. Although orthodontic consideration is discussed, this is very brief and gives minimal detail.

The subsequent parts three, four and five are divided by their anatomical parts: orbit, nose, maxilla and mandible. These vast parts discuss procedures such as fracture management, osteotomy procedures and free flap surgery for mandible reconstruction. These parts discuss orthodontic pre- and post-operative considerations in more detail. Part six consists of plastic surgery related to ear reconstruction. The final part is related to cleft lip and palate repair procedures. This is divided into sections based on classification of the cleft lip and palate deformity.

Some more common examples of craniofacial surgery were missed. For example, the book fails to mention mandible fracture management. It also doesn't account for inter-unit variation. Differences may arise in equipment available and surgical techniques. The writers are all based in the US, which may limit generalisability to practice for UK-based surgical trainees. However, the overriding principles are relevant to all surgical procedures with detailed illustrations and images. The online availability and video examples allow this book to be accessible at times when trainees may not have the hardback to hand, making this atlas a great addition to the repertoire of books required for trainees.

Claire Graham

\section{Here has been \\ an advertisement.}

\section{SPRINGERNATURE}

